This is the accepted version of the article published as "Scanlon, James J. and Wren, Stephen (2020) Unearthing novel thiazolidinone building blocks as carboxylic acid bioisosteres. Future Medicinal Chemistry, 12(20), pp. 1855-1864. ISSN (print) 1756-8919"

The version of record can be accessed at https://doi.org/10.4155/fmc-2020-0192 


\title{
Unearthing Novel Thiazolidinone Building Blocks as Carboxylic Acid Bioisosteres
}

\author{
James J. Scanlon and Stephen P. Wren * \\ Department of Chemical and Pharmaceutical Sciences, Faculty of Science, Engineering and Computing, Kingston University \\ London, Penryhn Road, Surrey, KT12EE. \\ *Author for correspondence: s.wren@kingston.ac.uk
}

\begin{abstract}
Aim: Thiazolidinones were prepared as building blocks for the replacement of carboxylic acids. Materials \& Methods: Chemical syntheses of thiazolidinones were developed. In addition, the drug-likeness of the target compounds were evaluated in silico. Results: The prepared compounds included the novel structure 4; 5-(3lodophenylmethylene)-2,4-thiazolidinedione. Conclusion: Exploration of the methods required to synthesise thiazolidinone building blocks was completed. This work allows future generation of bioisosteric analogues of drugs.
\end{abstract}

\begin{abstract}
The presence of carboxylic acid moieties in drugs can present disadvantages such as low passive diffusion across biomembranes. A carboxylic acid may be substituted with a suitable bioisostere, for example a thiazolidinedione (TZD), in order to avoid such adverse effects. The in silico pharmacokinetic properties for TZD building blocks were evaluated. The novel precursor $\mathbf{4}$ was characterised by spectroscopy and microanalysis confirmed its molecular structure. Preliminary investigations of subsequent reduction of alkene conjugation and Suzuki-cross coupling reactions were attempted without success. Nonetheless, integrating 2-MeTHF presents a novel protocol for the lithium borohydride reduction of benzylidene TZDs. The methods presented have the potential to translate to the coupling of real drug motifs to TZD containing motifs.
\end{abstract}

Keywords: bioisostere, thiazolidinone, carboxylic acid, building blocks.

The carboxylic acid moiety is present in many endogenous substances like amino acids, triglycerides and prostanoids and plays a cardinal role in drug design. This functional group is part of a diverse family of drugs, exemplified by the fact that a significant number $(>450)$ of marketed drugs are carboxylic acid containing molecules [1a]. Example drugs include nonsteroidal anti-inflammatory drugs (NSAIDs), $\beta$-lactam antibiotics [2], anticoagulants such as aspirin (which contains salicylic acid) and the eczema/dermatitis treatment betafucin (which contains fusidic acid) [3]. Nevertheless, despite the success of some carboxylic acid-containing drugs, the presence of this polar moiety can often be a disadvantage [4]. For instance, carboxylic acids can confer a limited ability to passively diffuse across lipid membranes such as those in the blood brain barrier and the intestine [5]. Moreover, metabolism of the carboxylic acid moiety (glucuronidation) can complicate pharmacotherapy, and its idiosyncratic toxicity may also present a problem [4]. A typical approach proposed by medicinal chemists, to circumnavigate such adverse effects, is the replacement of a carboxylic acid functional group with a suitable surrogate or bioisostere [1a, 4]. In general, bioisosterism is a concept defined by how a set of molecules exhibits physical and chemical similarities in relation to their biological interactions [6]. Judicious replacement of the carboxylic acid moiety is deemed successful if similarities exist between bioisosteres, coinciding with subtle modifications in order to improve levels of toxicity, stability, selectivity and/or absorption [4].

As part of our eventual objective of assembling 96-well plates of proprietary building blocks featuring bioisosteres (see Figure 1), we intended to investigate the late-stage chemical attachment of building blocks to drug motifs for the production of proof-of-concept compounds. We envisaged use of an appropriate synthetic handle (aryl halide) could facilitate such chemistry. 


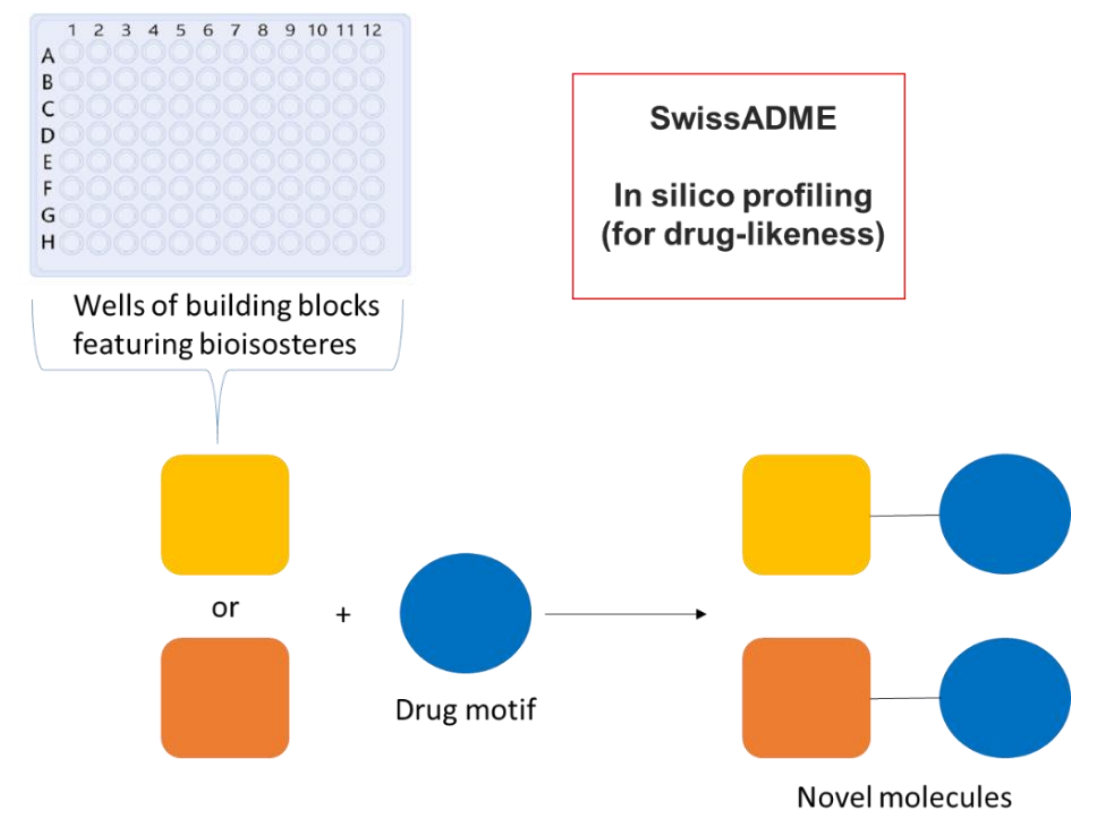

Figure 1: Generation of a bioisostere building block 'toolbox'

The construction of a versatile range of biaryls has been reported via the palladium-catalysed Suzuki cross coupling reaction using aryl halides [7, 8]. An example PPAR $\gamma /$ FFAR1 dual agonist (1) belonging to this family with a thiazolidinedione (TZD) group at one terminus is depicted in Figure 2 [1b]. Moreover, compounds comprising these substructures constitute important building blocks in organic synthesis and medicinal chemistry [9]. We explored this approach to construct model adducts (Figure 4) as a first foray into reaching our objective of translating proofof concept methods to the coupling of real drug motifs to TZD-containing motifs (using molecular fragments bearing the appropriate functionality) in mind.<smiles>O=C1NC(=O)C(Cc2ccc(OCc3cccc(-c4ccccc4)c3)cc2)S1</smiles>

Figure 2: Compound 1 (bearing a biphenyl motif)

The aim of the methodologies proposed herein was to afford a reliable 'toolbox' necessary in establishing a catalogue of building blocks. In addition, the present work aimed to develop building blocks featuring a suitable bioisostere of the carboxylic acid moiety, thus allowing the pursuit of drug analogues. The chemical structures within this paper resemble the NSAID Felbinac (biphenylylacetic acid) and replacement of the carboxylic acid of Felbinac and related derivatives may be of interest to the wider community. As an aside, one of the phenyl rings of Felbinac has been replaced with a BN bioisostere using rhodium-catalysed arylation chemistry [10]. TZDs (also termed glitazones) have been shown to reduce insulin resistance [11], concurring with a rise in glucose disposal and reduced hepatic glucose production. TZDs are featured in the family of anti-diabetic drugs, used in the management of type 2 diabetes (e.g. pioglitazone and rosiglitazone; Figure 3). Members of the glitazone structural class stimulate the activation of the peroxisome proliferator activated receptor $\gamma$ (PPAR $\gamma$ ) [11]. Comparably, a diverse group of carboxylic acid-containing compounds (as drug-like candidates) have also been shown to stimulate the activation of PPARY [12]. Furthermore, TZDs confer moderate acidity $(p K a=6-7)$ and relative lipophilicity [13]. This makes the TZD group prone to augmenting drug permeability and diffusion across biological membranes relative to corresponding carboxylic acids. Hence, by the bioisostere principle, TZD is a suitable surrogate. The utility of this 
strategy is already evident, for the TZD bioisostere has resulted in a broad range of important therapeutic agents [1].

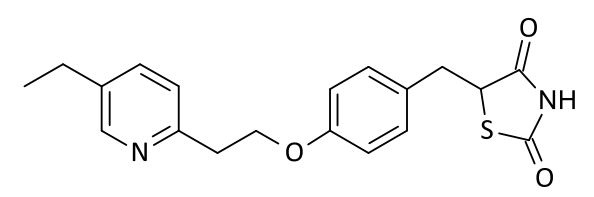

(a)<smiles>CN(COc1ccc(CC2SC(=O)NC2=O)cc1)c1ccccn1</smiles>

(b)

Figure 3: PPARY agonists pioglitazone (a) and rosiglitazone (b)

We used the free in silico tool SwissADME [14] to predict ADME parameters and pharmacokinetic properties of the target molecules described herein. Accordingly, the in silico properties of target TZD building blocks, in conjunction with their corresponding carboxylic acids, were profiled in an effort to prioritise our synthetic efforts [15]. In this context, the physicochemical assessment of each building block dealt only with predicted permeability as barriers to absorption in relation to its suitability. Thus, as highlighted by Lipinski, a building block with a mass $\leq 450 \mathrm{Da}, \log \mathrm{P}$ $<5,<5 \mathrm{H}$-bond donors and $<10 \mathrm{H}$ bond acceptors predicted that a drug is likely to be orally active. Moreover, Ertl established the desirable topological suitable polar surface area parameters (TPSA) for efficient permeability across membranes (blood brain barrier: 60-70 $\AA^{2}$; cell membranes > $140 \AA^{2}$ ) [16].

Our initial experiments centred on the preparation of halobenzylidene thiazolidinediones (BTZDs) [17a-e], bearing appropriate substitution on the aromatic ring. Access to novel structures is a constant goal of ours, however, we reasoned that synthesis of known compounds was also useful for method development. Several methods have reported on the synthesis of BTZDs; generally, these have followed a Knoevenagel or aldol condensation approach. Example literature findings disclose the use of piperidine and acetate in toluene [18], polyethylene glycol-300 [19], and sodium acetate in acetic acid [20]. Consequently, each of these methods suffer from several disadvantages such as drawn out work-up procedures, use of toxic solvents and/or requirement of special apparatus. To overcome these drawbacks, a solvent-free reaction procedure, using ethylenediamine and acetic acid, has been proposed [21, 22]. Subsequently, the involvement of this Brönsted acid-base catalyst holds many advantages: the exclusion of toxic reagents; lower impact on the environment; and simplicity in process and handling. We describe the synthesis of BTZDs by the Knoevenagel condensation of aromatic aldehydes with TZD in the presence of ethylenediamine and acetic acid. Relatively little information exists concerning the effectiveness of this approach regarding aryl halides. To help address these gaps, we explored novel reaction schemes involving 3-iodobenzaldehyde and 4bromobenzaldehyde with TZD (Figure 5). In addition, we investigated a literature aldol condensation scheme, for the purpose of cross-evaluating reaction schemes [31].

\section{Experimental}

\section{Reagents \& Equipment}

Reagents, solvents and catalysts were purchased from Sigma Aldrich (Gillingham, Dorset, UK) and were used without further purification. The hotplate magnetic stirrer used was supplied by Camlab (MS-H280-Pro). TLC analyses were performed using aluminium backed silica gel coated plates $\left(60 \mathrm{~F}_{254}\right)$ and developed using UVGL-58 Handheld UV Lamp (254-365nm). Flash chromatography was performed using Davisil ${ }^{\bullet}$ chromatography grade silica (pore size 60 angstrom, particle size 35-70 micron). Melting points were determined on a Gallenkamp (electronic) apparatus. IR spectra were recorded on a Nicolet iS5 FTIR spectrometer, with the Thermo Scientific ${ }^{\text {TM }}$ iD7 ATRdiamond accessory included. NMR spectra were recorded on an Oxford NMR ADVANCE II instrument operating at $400 \mathrm{MHz}$ for ${ }^{1} \mathrm{H}$ and ${ }^{13} \mathrm{C} N M R$, and were performed in DMSO- $\mathrm{d}_{6}$ solutions (signals are recorded as $\delta / p p m$ ). Mass spectra was recorded on a Sciex API 3000 machine. Microanalysis was performed by Medac Ltd. (Chobham, Surrey, UK). 


\section{Synthesis}

The reaction schemes shown in Figure 4 illustrate our efforts to prepare 4-8 and $\mathbf{9 a - b .}$<smiles>[X]c1ccc(C=O)cc1[X]</smiles>

2<smiles>O=C1CSC(=O)N1</smiles>

3<smiles>C#CC#C</smiles>

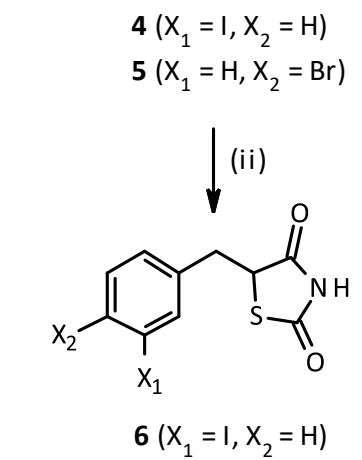

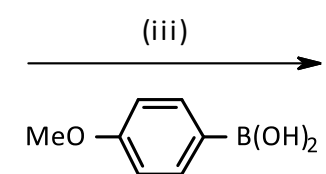<smiles>[R]c1ccc(/C=c2\sc(=O)[nH]c2=C)cc1[R]</smiles>

$$
7\left(\mathrm{R}_{1}=4-\mathrm{MeOC}_{6} \mathrm{H}_{4}, \mathrm{R}_{2}=\mathrm{H}\right)
$$$$
8\left(R_{1}=H, R_{2}=4-\mathrm{MeOC}_{6} H_{4}\right)
$$<smiles>[R]c1ccc(CC2SC(=O)NC2=O)cc1[R]</smiles>

$$
\text { 9a }\left(\mathrm{R}_{1}=4-\mathrm{MeOC}_{6} \mathrm{H}_{4}, \mathrm{R}_{2}=\mathrm{H}\right)
$$$$
9 b\left(R_{1}=H, R_{2}=4-M_{e O C} H_{4}\right)
$$

(i) Synthesis of 4-5 using PhMe, NaOAc, $180^{\circ} \mathrm{C}$, molecular sieves or ethylenediamine, $\mathrm{AcOH}, 80^{\circ} \mathrm{C}$ (16-42\% yield; see Table 2). (ii) Attempted conversion of 4 (to 6) using $\mathrm{LiBH}_{4}, 2-\mathrm{MeTHF}$, pyridine, $110^{\circ} \mathrm{C}$ gave no reaction. (iii): Attempted conversion of 4 (to 7)and 5 (to 8) using either 4-Methoxyphenylboronic acid, $\mathrm{K}_{2} \mathrm{CO}_{3}$, $\mathrm{Pd} / \mathrm{C}, \mathrm{H}_{2} \mathrm{O}$, RT or 4-Methoxyphenylboronic acid, $\mathrm{K}_{2} \mathrm{CO}_{3}$ and either $\mathrm{Pd} / \mathrm{C}, \mathrm{H}_{2} \mathrm{O}, \mathrm{RT}$ or $\mathrm{Pd}(\mathrm{OAc})_{2}$, $\mathrm{PhMe}, 90^{\circ} \mathrm{C}$ gave no reaction. (iv) Future reactions.

Figure 4: Summary of synthetic attempts to access TZD building blocks: (i) condensation reaction; (ii) reduction; (iii) cross coupling reaction.

\section{Results \& Discussion}

Determination of predicted physiochemical/ADME properties

The SwissADME application [14] enabled the swift identification of appropriate drug-like building blocks. Early evaluation of the ADME parameters (Adsorption, Distribution, Metabolism and Excretion) may reduce the proportion of unsuccessful molecules resulting from pharmacokinetics-related issues.

The results from a study of conceivable building blocks are depicted in Table 1. As demonstrated, a TZD surrogate can lead to considerable variations in physiochemical properties in relation to the carboxylic acid moiety. The criteria set by Lipinski and Ertl, aided our selection of molecules with drug-like character. As shown in Table 1, the TZD containing building blocks exhibit greater $\log P_{\mathrm{o} / w}$ values than their carboxylic acid isosteres (compare entries ii and iv with i and iii). Correspondingly, the increased lipophilicity of a molecule will increase its value of $\log P_{\mathrm{o} / \mathrm{w}}$. These properties imply that the more polar carboxylic acid moiety can display relatively high-water solubility, an important characteristic for a drug-like candidate. Nonetheless, these aspects may also be linked to a compound's limitations [23]. A key possible deficit of the carboxylic acid moiety, is attributed to its short half-life (if metabolic turnover is relatively rapid). The limitation of a short half-life may be attributed to low $\log P_{\mathrm{o} / \mathrm{w}}$ by the associated promotion of rapid metabolic conversion (glucuronidation). This may induce serious adverse side effects, caused by the covalent modifications of proteins, succeeding from the metabolites (acyl glucuronides) [23].

However, an elevated value of the $\log P_{\mathrm{o} / \mathrm{w}}$ value will decrease the aqueous solubility of the compound (TZDs). Hansch and Leo proposed that highly lipophilic molecules will be partitioned and retained at the lipid interior of the cell membrane [24]. Hence, this may lead to the poorer absorption of drug candidates. TPSA is described as the sum of molecular surface areas arising from polar nitrogen and oxygen atoms together with their attached hydrogen atoms [16]. Such forces are involved as a molecule approaches the polar surface of a cell membrane. As the TZD surrogate presents an innately higher TPSA than its carboxylic acid isostere (see compounds 10a-b in entries 
ii and iv of Table 1), it is desirable that each building block be only minimally functionalised. By this criteria, entries i and iii (compounds $9 \mathbf{a}-\mathbf{b}$, Table 1) present biaryl scaffolds that are deemed unsuitable for possible BBB penetration. Nonetheless, permeability of the cell membrane is still predicted to be viable as the TPSA values of TZDs depicted in entries i and iii (Table 1) do not exceed $140 \AA^{2}$ [25]. Consequently, binding to the PPAR receptor (found primarily in adipose tissue) could be possible, enabling the TZD-containing compounds to exert a glucose lowering effect [26]. The scaffold of the building block must not disrupt the core physicochemical properties of the TZD surrogate. A short aliphatic spacer was deemed desirable in order to minimise the possible aqueous solubility deficiency caused by the conjugation of the biaryl scaffold (compare 9a-b with compounds 7-8).

Table 1: Summary of results gained using SwissADME for various properties of TZD- and carboxylic acid-containing biaryl building blocks. The relative molecular mass (RMM, g.mol ${ }^{-1}$ ), topological polar surface area (TPSA, $\AA^{2}$ ), $\log P_{\mathrm{o} / w}$, and permeability across the blood brain barrier (BBB) are highlighted.

\begin{tabular}{|c|c|c|c|c|c|c|}
\hline Entry & Building block & $\mathrm{RMM}$ & TPSA & Consensus $\log P_{0} / \mathrm{w}^{\mathrm{a}}$ & BBB permeant & Lipinski \\
\hline $\mathrm{i}$ & 9a (see Figure 4) & 313 & 80.7 & 2.97 & No & 0 violations \\
\hline ii & & 258 & 49.7 & 2.87 & Yes & 0 violations \\
\hline iii & $9 \mathbf{b}$ (see Figure 4) & 313 & 80.7 & 2.97 & No & 0 violations \\
\hline iv & & 258 & 49.7 & 2.87 & Yes & 0 violations \\
\hline
\end{tabular}

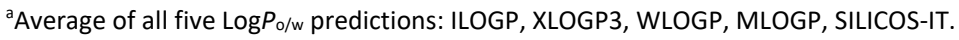

\section{Establishing the building blocks}

The versatility of Suzuki cross coupling reactions between aryl boronic acids and aryl halides, in the presence of a palladium catalyst, has been well demonstrated [7, 27-29]. Comparably, such organoboron reagents exhibit greater functional group compatibility than their organo-metal counterparts for instance, organozinc and Grignard reagents [30]. Monosubstituted phenylboronic acids are commonly inexpensive and their innocuous nature serves to provide benefits such as low toxicity and high stability. Moreover, the mode of reaction can be conducted in an aqueous media, which provides economic and environmental advantages [28]. Ortho- and para- substitution of aryls have been thoroughly examined, however meta-selective $\mathrm{C}-\mathrm{H}$ functionalization has been less explored [29]. We reasoned that substitution at the meta position could be significant in expanding the catalogue of TZD building blocks and subsequent drug candidates (see 7, for example, in Figure 4).

Furthermore, reduction of the alkene present in 4-5 would give rise to 9a-b (Figure 4). Giles et al. proposed a suitable reaction scheme for this process that incorporated use of lithium borohydride in $\mathrm{THF} /$ pyridine. Their procedure afforded a high yield with relatively short reaction time, demonstrating 


\section{Article Body Template}

the high selectivity of the reagent as a BTZD reducing agent [31]. Critically, the procedure proposed by Giles was deemed to be not in keeping with our 'green chemistry' philosophy. We envisaged that the commercially available solvent 2-methylTHF, afforded from renewable resources, presents an appropriate modification to this reduction process [32].

The results from our efforts to prepare halobenzyl thiazolidinediones (in 16-42\% yield) are summarised in Table 2. From the corresponding halo-benzaldehyde (2) and TZD (3), 4 was prepared using a modified form of the reported method by Giles et al. [31]. The condensation process was carried out using sodium acetate in refluxing toluene but, in our hands, no identifiable products were isolated using this reaction temperature. In an endeavour to assist the aldol condensation, acetic acid was incorporated into the reaction mixture at $180^{\circ} \mathrm{C}$ [30]. Molecular sieves were included to remove any subsequent water production. In accordance with Le Chatelier's Principle, this protocol should increase the rate of the forward reaction process, yielding product 4 . This procedure presented a conceivable one-pot means of procuring precursors of our desired building blocks. An example ${ }^{1} \mathrm{H}$ NMR diagnostic observation was the singlet at $\delta 7.73 \mathrm{ppm}$ illustrating the formation of the carboncarbon double bond present in compound 4.

Table 2: Preparation of halobenzyl thiazolidinediones

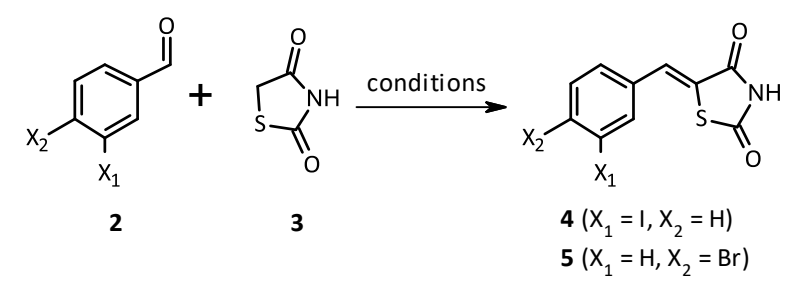

\begin{tabular}{ccccc}
\hline Entry & $\mathrm{X}^{1}$ & $\mathrm{X}^{2}$ & Time for condensation (h) & $\begin{array}{c}\text { Yield of 4-5 } \\
(\%)\end{array}$ \\
\hline a & I & H & 6 & $16^{\text {a }}$ \\
b & I & H & 1.5 & 29 \\
c & H & $\mathrm{Br}$ & 2.0 & N.R. \\
d & H & $\mathrm{Br}$ & 64 & 42 \\
e & I & H & 64 & 28
\end{tabular}

a: PhMe, $\mathrm{NaOAc}, 180^{\circ} \mathrm{C}$, molecular sieves; b-e: Ethylenediamine, $\mathrm{AcOH}, 80^{\circ} \mathrm{C}$ (N.R.: No reaction. ${ }^{\text {aY }}$ ield of crude 4).

In contrast to the aldol condensation-mediated production of $\mathbf{4}$ we found other Knoevenagel condensations proceeded smoothly to produce 4-5. The quantity of catalyst and reaction time had important effects on the reaction. In accordance with Zhang and Zhou [22], 80 ${ }^{\circ} \mathrm{C}$ was chosen as the reaction temperature. Ethylenediamine and acetic acid were employed for the synthesis of 4 and, after 1.5 hours, the product precipitated out of solution (entry 2, Table 2).

Next, we attempted to transpose these reaction conditions to the synthesis of $\mathbf{5}$. However, the initial practice involving para-bromo benzaldehyde gave rise to various complications. In contrast to 4, TLC analysis identified the formation of two products and attempted recrystallisation (using ethyl acetate and hexane) or flash chromatography did not aid purification. As such, the reaction procedure used 


\section{Article Body Template}

for the synthesis of compound $\mathbf{5}$ could not be emulated for the formation of $\mathbf{4}$ (suggesting the starting aldehydes react differently). We modified the procedure (to avoid tedious extraction procedure) by prolonging the reaction time. Moreover, the concentration of both ethylenediamine was increased and the quantity of acetic acid reduced (keeping the amount of 4-bromobenzaldehyde constant at $3.21 \mathrm{mmol}$ ). Thus, a greater yield of pure 5 , with an observed melting point range of $242-244^{\circ} \mathrm{C}$ (Found: $252-253^{\circ} \mathrm{C}$; [31]) was obtained. This effective methodology was emulated in order to yield the novel iodide 4. Despite the prolonged reaction time, partnered with the altered concentrations of ethylenediamine and acetic acid, there was no increase in yield. Spectroscopic and microanalysis confirmed the formation of the novel iodo-BTZD 4.

The methodology used for the attempted regioselective reduction of $\mathbf{4}$ to $\mathbf{6}$ was adapted from Giles et al [31]; the inclusion of pyridine was reported to modify the reactivity of the $\mathrm{LiBH}_{4}$, to selectively reduce the alkene function. Accordingly, 4 was dissolved in dry 2-MeTHF and the incorporation of this solvent, as a substitute for THF, is well founded. 2-MeTHF presents a more stable solvent than THF during lithiation reactions [32] and, as such, 2-MeTHF offers a sustainable/eco-friendly alternative to THF. Thus, the reaction was ceased, after refluxing for $6 \mathrm{hrs}$, as per the literature [33] but ${ }^{1} \mathrm{H}$ NMR analysis after work-up revealed that the starting material was returned (Figure 4). We are currently investigating the synthesis of $\mathbf{9 a - b}$ from derivatives 7-8 (Figure 4).

We intended to investigate a late stage functionalisation of aryl halides using Suzuki-type conditions in the presence of TZD sulfur atoms. A similar strategy involving early-stage Suzuki coupling between a sulfur-containing TZD unit and meta-bromide is precedented [34]. Our attempted synthesis of 7-8 was adapted from Sakurai's methodology for the room temperature generation of BTZDs [28]. Palladium on carbon offers an efficient, ligand free and heterogenous catalysed protocol for the coupling of 4-5 and boronic acids. The premise of incorporating $\mathrm{Pd} / \mathrm{C}$ is founded on its stability in open air/water and to base, often not requiring solvent degassing or an inert atmosphere. After $\mathbf{4}$ and $\mathbf{5}$ were reacted with 4-methoxyboronic acid in the presence of $\mathrm{K}_{2} \mathrm{CO}_{3}$ and $\mathrm{Pd} / \mathrm{C}$, at room temperature, no ${ }^{1} \mathrm{H}$ NMR signals consistent with the desired products were present after analysis of the crude mixtures and starting material was returned in each case (see Table 3). Also, attempted Suzuki coupling between 5 and 4-methoxyphenylboronic acid in the presence of $\mathrm{Pd}(\mathrm{OAc})_{2}$ and hot toluene did not yield the desired product, 8. Further studies here could include the use of prolonged heating and the incorporation of varying solvents, to optimise the Suzuki cross-coupling scheme [1]. Earlier incorporation of the aryl group prior to condensation with TZD may also be worth exploration in case the sulfur affects the catalyst [35]. However, evidence of Suzuki couplings in TZD-containing systems has been reported [34].

Table 3: Attempted palladium cross-coupling reactions 


\section{Article Body Template}

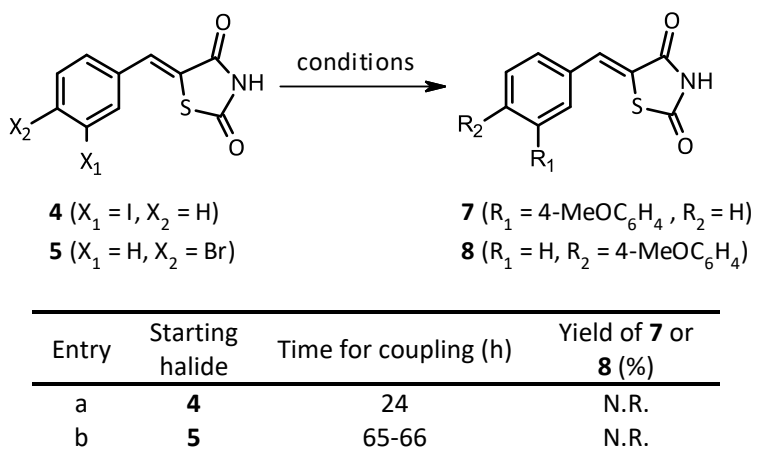

a: 4-Methoxyphenylboronic acid, $\mathrm{K}_{2} \mathrm{CO}_{3}, \mathrm{Pd} / \mathrm{C}, \mathrm{H}_{2} \mathrm{O}$, RT. b: 4-Methoxyphenylboronic acid, $\mathrm{K}_{2} \mathrm{CO}_{3}$ and either $\mathrm{Pd} / \mathrm{C}, \mathrm{H}{ }_{2} \mathrm{O}, \mathrm{RT}$ or $\mathrm{Pd}(\mathrm{OAc})_{2}, \mathrm{PhMe}$, $90^{\circ}$ C. N.R.: No reaction.

\section{Conclusions}

In summary, our building block in silico studies suggested that the TZD replacement for a carboxylic acid presented no pharmacokinetic violations following the guidelines set by Lipinski. However, bloodbrain barrier penetration may not viable for compounds 9a-b; Ertl [16] describes that permeability is permitted when TPSA is lower than $80 \AA^{2}$. Nonetheless, as the abundance of PPARY is stored primarily within adipose tissue, cell membrane permeability would be required for treatments of metabolic disease and, hence, the building blocks 7-8 adhere to the enclosed guidelines (TPSA $<140 \AA^{2}$ ). Our reaction schemes were aimed at presenting a facile and environmentally benign means of obtaining each building block. It has been shown, that ethylenediamine and acetic acid can be used as a catalytic alternative to the aldol condensation of sodium acetate and acetic acid (4), for the preparation of BTZDs. Minimal purification steps, recyclable amine catalyst and higher yields are the core benefits presented by this protocol and the formation of the novel compound $\mathbf{4}$ was confirmed. Moreover, the para substituted alternative was also obtained (5). However, the optimum amine concentration remains to be established and, in addition, further investigation could ascertain the ideal reaction conditions for each benzaldehyde. A novel solvent application of 2-MeTHF was explored, during the lithiation reduction attempt of a BTZD. Despite the limitations presented in the enclosed experimental (return of starting material), our studies around the synthetic utility of 2-MeTHF are continuing in combination with lithium borohydride. It has been shown to be an effective solvent for low temperature lithiation reactions [32].

Both heterogenous and homogenous palladium catalysed reactions were explored, for the attachment of a phenyl ring onto a TZD scaffold via a 'synthetic handle'. Further studies in this area will be reported on in due course. Optimisation of catalyst loading in conjunction with a less viscous solvent (such as DMAc), at a higher temperature, may yield the necessary rate of diffusion for a successful catalytic process.

\section{Future perspective}

The methods presented have the potential to translate to the coupling of real drug motifs to TZD containing motifs. In other words, potential (larger) drug molecules could be assembled using building blocks such as 4 . Further refinement of chemical methodology is in progress in our laboratory for the 


\section{Article Body Template}

application to real carboxylic acid-containing drug candidates and storage of solutions of building blocks in a 96-well plate library format. The construction of biaryls, as illustrated in Figure 4, generated examples of model building blocks. Although the disclosed methodologies evaluate the synthesis of biaryl building blocks, the reaction schemes illustrated are not limited to this role. In future, more suitable drug like candidates could be viably established and we plan to explore how their biological/ADME profiles compare to reference drugs such as pioglitazone. In terms of in silico perspectives, it seems reasonable that such molecules could stabilize the ligand binding domain of PPAR $\gamma$ given that the binding modes of TZDs are well conserved [36].

\section{Acknowledgments}

We thanks Ms Tara Brodie (for mass spectrometry) Mr Mahboub Merzouk (NMR assistance), Mr Owen Lawler (technical support) and both Ms Amal Takar and Ms Sarah Namuk (for background literature searching and additional, unpublished, experiments).

\section{Supplementary Information}

See attached file.

\section{Financial \& competing interests disclosure}

The authors have no other relevant affiliations or financial involvement with any organisation or entity with a financial interest or financial conflict with the subject matter or materials discussed in the manuscript apart from those disclosed. No writing assistance was utilised in the production of this manuscript.

\section{Summary Points}

\section{Experimental section}

- Chemical routes for the synthesis of thiazolidinones were investigated.

- The drug-likeness of the target compounds were evaluated in silico.

Results \& discussion

- A set of TZD building blocks, including novel iodide 4, were prepared with the intention of replacing carboxylic acid motifs.

\section{Conclusion}

- Exploration of the synthetic methods studied will allow for future generation of bioisosteric analogues of real drug molecules. Novel building blocks are currently being assembled in our laboratory.

\section{References}

1. (a) Lassalas P, Gay B, Lasfargeas C, James MJ, Tran V, Vijayendran KG, Brunden KR, Kozlowski MC, Thomas CJ, Smith AB 3rd, Huryn DM, Ballatore C. Structure Property Relationships of Carboxylic Acid Isosteres. J. Med. Chem., 59(7), 3183-3203 (2016).* A comprehensive study of bioisosteres. (b) Darwish KM, Salama I, Mostafa S, Gomaa MS, Helal MA. Design, synthesis, and biological evaluation of novel thiazolidinediones as PPAR $\gamma /$ FFAR1 dual agonists. Eur. J. Med. Chem., 109, 157172 (2016).

2. Kong K, Schnepper L, Mathee K. Beta-lactam antibiotics: from antibiosis to resistance and bacteriology. J. Path. Microbiol. Immunol., 1, 1-36 (2010).

3. Girolomoni G, Mattina A, Manfredini S, Vertuani S, Fabrizi G. Fusidic acid betamethasone lipid cream. Int. J. Clin. Prac., 70 (S184), 4-13 (2016).

4. Ballatore C, Huryn DM, Smith AB 3rd. Carboxylic Acid (Bio)Isosteres in Drug Design. ChemMedChem, 8(3), 385-395 (2013).

** this review summarises commonly employed carboxylic acid bioisosteres and how they are used in drug design. 


\section{Article Body Template}

5. Pajouhesh H, Lenz GR. Medicinal Chemical Properties of Successful Central Nervous System Drugs. NeuroRx, 2(4), 541-553 (2005).

6. Ali G, Islam NUI, Subhan F, Khan I. Input of Isosteric and Bioisosteric Approach in Drug Design. J. Chem. Soc. Pak., 36(5), 120 (2014).

7. Martin R, Buchwald SL. Palladium-Catalyzed Suzuki-Miyaura Cross-Coupling Reactions Employing Dialkylbiaryl Phosphine Ligands. Acc. Chem. Res., 41(11), 1461-1473 (2008).

8. Diez AS, Graziano-Mayer M, Radivoy G, Volpe MA. Suzuki-Miyaura cross-coupling of aryl iodides and phenylboronic acid over palladium-free $\mathrm{CeO}_{2}$ catalysts. Appl. Cat. A: Gen., 482, 24-30 (2014).

9. Liu B, Moffett KK, Joseph RW, Dorsey BD. Efficient synthesis of substituted biaryl anilines and biaryl phenols via a Suzuki cross-coupling reaction. Tet. Lett., 46(11), 1779-1782 (2005).

10. Rudebusch GE, Zakharov LN, Liu S-Y. Rhodium-Catalysed Boron Arylation of 1,2-Azaborines. Angew. Chem. Int. Ed. Engl., 52(35), 9316-9319 (2013).

11. Quinn CE, Hamilton PK, Lockhart CJ, McVeigh GE. Thiazolidinediones: effects on insulin resistance and the cardiovascular system. Brit. J. Pharmacol., 153(4), 636-645 (2007).

12. Henke BR, Blanchard SG, Brackeen MF, Brown KK, Cobb JE, Collins JL, et al. N-(2-Benzoylphenyl)-I-tyrosine PPARY Agonists. 1. Discovery of a Novel Series of Potent Antihyperglycemic and Antihyperlipidemic Agents. J. Med. Chem., 41(25), 5020-5036 (1998).

13. Lipinski CA, Fiese EF, Korst RJ. pKa, Log P and MedChem CLOGP Fragment Values of Acidic Heterocyclic Potential Bioisosteres. Quant. Struct.-Act. Relat., 10, 109-117 (1991). * An important contribution to the bioisostere field.

14. Daina A, Michielin O, Zoete V. SwissADME: a free web tool to evaluate pharmacokinetics, drug-likeness and medicinal chemistry friendliness of small molecules. Sci. Rep., 7(42717), 1-13 (2017).

15. Lipinski CA, Lombardo F, Dominy BW, Feeney PJ. Experimental and Computational Approaches to Estimate Solubility and Permeability in Drug Discovery and Development Settings. Adv. Drug Del. Rev., 46 (1-3), 3-26 (2001). * A highly useful study of aspects of measuring drug-likeness.

16. Ertl P, Rohde B, Selzer P. Fast calculation of molecular polar surface area as a sum of fragment-based contributions and its application to the prediction of drug transport properties. J. Med. Chem., 43(20), 3714-3717 (2000). * A paper of significance for the medicinal chemist interested in polar surface area.

17. (a) Yasmin S, Capone F, Laghezza A, Dal Piaz F, Loiodice F, Vijayan V, Devadasan V, Mondal SK, Atlı Ö, Baysal M, Pattnaik AK, Jayaprakash V , Lavecchia, A. Novel Benzylidene Thiazolidinedione Derivatives as Partial PPARY Agonists and their Antidiabetic Effects on Type 2 Diabetes. Sci. Rep., 7, 14453 (2017); (b) Meta-iodide 4: no references found in Scifinder (as of $8^{\text {th }}$ June 2020); only 7 commercial suppliers (for example, Aurora, FCH); (c) Para-iodo analogue of compound 4 is reported in Scifinder - see: Cho, Hun; Lee, Yeon Ung (Industry-Academic Co-op Found Chosun Univ, Republic of Korea). Composition for destructing micro-algae. KR2016126772 (2016).; (d) Para-bromide 5 is described in many Scifinder references, for example: Geldenhuys WJ, Funk MO, Van der Schyf CJ, Carroll RT. A scaffold hopping approach to identify novel monoamine oxidase B inhibitors. Bioorg. Med. Chem. Lett., 22(3), 1380-1383 (2012); (e) Lu J, Lei L, Huan Y, Li Y, Zhang L, Shen Z, Hu W and Feng Z. Design, Synthesis, and Activity Evaluation of GK/PPAR $\gamma$ Dual-Target-Directed Ligands as Hypoglycemic Agents. ChemMedChem, 9, 922 - 927 (2014).

18. Zidar N, Tomašić T, Šink R, Rupnik V, Kovač A, Turk S. Discovery of Novel 5-Benzylidenerhodanine and 5Benzylidenethiazolidine-2,4-dione Inhibitors of MurD Ligase. J. Med. Chem., 53(18), 6584-6594 (2010).

19. Mahalle SR, Netankar PD, Bondge SP, Mane RA. An efficient method for Knoevenagel condensation: a facile synthesis of 5-arylidenyl 2,4-thiazolidinedione. Green Chem. Lett. Rev., 1(2-4), 103-106 (2007). * The authors feel this work is a significant contribution to the area of TZD synthesis.

20. Sapkal SB, Shelke KF, Kategaonkar AH, Shingare MS. Dual role of ammonium acetate for solvent-free synthesis of 1,3disubstituted-2,3-dihydro-1H-naphth-[1,2e] [1,3]-oxazine. Green Chem. Lett. Rev., 2(2), 57-60 (2009).

21. Gandini A, Bartolini M, Tedesco D, Martinez-Gonzalez L, Roca C, Campillo NE, et al. Tau-Centric Multitarget Approach for Alzheimer's Disease: Development of First-in-Class Dual Glycogen Synthase Kinase 3B and Tau-Aggregation Inhibitors. J. Med. Chem., 61(17), 7640-7656 (2018).

22. Zhang Y, Zhou Z. A Solvent-Free Protocol for the Green Synthesis of 5-Arylidene-2,4-thiazolidinediones Using Ethylenediamine Diacetate as Catalyst. Org. Chem. Int., 33(10), 2220-2225 (2012).

23. Bailey MJ, Dickinson RG.. Acyl glucuronide reactivity in perspective: biological consequences. Chem. Biol. Interact., 145(2), 117-137 (2003).

24. Hansch C, Björkroth JP, Leo A. Hydrophobicity and central nervous system agents: on the principle of minimal hydrophobicity in drug design. J. Pharm. Sci., 76(9), 663-687 (1987).

25. Matsson P, Kihlberg J. How big is too big for cell permeability? J. Med. Chem., 60(5), 1662-1664 (2017).

26. Guan Y, Hao C, Cha DR, Rao R, Lu W, Kohan DE. Thiazolidinediones expand body fluid volume through PPARgamma stimulation of ENaC-mediated renal salt absorption. Nat. Med., 11(8), 861-866 (2005). 


\section{Article Body Template}

27. Littke AF, Dai C, Fu GC. Versatile Catalysts for the Suzuki Cross-Coupling of Arylboronic Acids with Aryl and Vinyl Halides and Triflates under Mild Conditions. J. Am. Chem. Soc., 22(17), 4020-4028 (2000).

28. Sakurai H, Tsukuda T, Hirao T. Pd/C as a Reusable Catalyst for the Coupling Reaction of Halophenols and Arylboronic Acids in Aqueous Media. J. Org. Chem., 67(8), 2721-2722 (2002).

29. Dey A, Agasti S, Maiti D. Palladium catalysed meta-C-H functionalization reactions. Org. Biomol. Chem., 14(24), 5440$5453(2016)$.

30. Fyfe, JWB, Watson AJB. Recent developments in organoboron chemistry: Old dogs, new tricks. Chem., 3(1), 31-55 (2017). 31. Giles RG, Lewis NJ, Quick JK, Sasse MJ, Urquhart MWJ, Latifa Youssef. Regiospecific Reduction of 5-Benzylidene-2,4Thiazolidinediones and 4-Oxo-2thiazolidinethiones using Lithium Borohydride in Pyridine and Tetrahydrofuran. Tetrahedron, 56(26), 4531-4537 (2000).

32. Aycock DF. Solvent Applications of 2-Methyltetrahydrofuran in Organometallic and Biphasic Reactions. Org. Proc. Res. Dev., 11(1), 156-159 (2006).

33. Negishi E, Hu Q, Huang Z, Qian M, Wang G. Palladium-Catalyzed Alkenylation by the Negishi Coupling. AldrichimicaACTA, 30(3), 71-86 (2005).

34. Chin AC, Holcomb R, Piatyszek MA, Singh U, Tolman RL, Akama T, Kanda Y, Asai, A, Yamashita Y, Endo K, Yamaguchi H. WO2001/002377 (Geron Corporation and Kyowa Hakko Kogyo Co Ltd). See conversion of compound 25 to compound 74 as described in the patent application).

35. Liu J, Kimmel K, Dao K, Liu Y, Qi M. Identification and Elimination of an Unexpected Catalyst Poison in Suzuki Coupling. Org. Process Res. Dev., 22, 111-116 (2018).

36. Lee MA, Tan L, Yang H, Im Y-G, Im YJ. Structures of PPARY complexed with lobeglitazone and pioglitazone reveal key determinants for the recognition of antidiabetic drugs. Sci. Rep., 7(16837), 1-11 (2017). 